Service social

\title{
Un logiciel d'intervention psychosociale, pourquoi pas?
}

\section{Claire Lalande-Gendreau et Françoise Turgeon-Krawczuk}

Volume 36, numéro 1, 1987

L'informatique dans les services sociaux

URI : https://id.erudit.org/iderudit/706342ar

DOI : https://doi.org/10.7202/706342ar

Aller au sommaire du numéro

\section{Éditeur(s)}

École de service social de l'Université Laval

\section{ISSN}

1708-1734 (numérique)

Découvrir la revue

\section{Citer cet article}

Lalande-Gendreau, C. \& Turgeon-Krawczuk, F. (1987). Un logiciel d'intervention psychosociale, pourquoi pas? Service social, 36(1), 96-110. https://doi.org/10.7202/706342ar

\section{Résumé de l'article}

Dans cet article sur la démarche d'informatisation du dossier social, les auteures procèdent à la description et à l'analyse de leur matériel. Le logiciel se compose d'une grille d'intervention familiale et sociale et d'une série de tests remplis par les parents. La grille d'intervention constitue la partie principale du logiciel et elle traduit l'essentiel de l'activité professionnelle dans le milieu médical : l'évaluation familiale et sociale et le plan d'intervention psychosociale.

Les auteures abordent ensuite la question de leur responsabilité dans l'intégration du logiciel professionnel aux programmations informatiques des deux établissements auxquels elles appartiennent : l'hôpital Sainte-Justine et le Centre de services sociaux du Montréal métropolitain. Se basant en partie sur les théories émises par Peters et Waterman, et sur leur expérience, elles énumèrent quelques dispositifs et stimulants d'action. 
LALANDE-Gendreau, Claire, travailleuse sociale au C.S.S. Montréal Métropolitain, Hôpital Sainte-Justine.

TURGEON-KraWCZUK, Françoise, travailleuse sociale au C.S.S. Montréal Métropolitain, Hôpital Sainte-Justine.

\title{
Un logiciel d'intervention psychosociale, pourquoi pas?
}

\author{
Claire Lalande-Gendreau \\ Françoise Turgeon-Krawczuk
}

"Car souvent pour faire un bon programme professionnel, il est plus important d'être compétent dans la profession visée qu'en informatique même. " ${ }^{1}$

Dans sa chronique " Demain l'An 2000 ", Yves Leclerc disait, qu'à sa connaissance, les programmes spécifiquement adaptés à une profession sont pratiquement introuvables. Travaillant depuis plus de deux ans sur l'informatisation des données psychosociales, nous avons pu vérifier cette affirmation en ce qui concerne la profession du service social. $\mathrm{Ce}$ fut une des raisons qui nous ont incitées à nous mettre à la tâche, devant l'éventualité de l'informatisation des cliniques spécialisées auxquelles nous appartenons.

Dans un article précédent ${ }^{2}$, nous avons décrit les premières étapes de cette démarche à partir de l'historique de la décision d'informatiser le dossier social clinique. La mise sur pied et le cheminement d'un comité ad hoc, à l'intérieur du Département de service social, sont examinés sous différents aspects : les attentes, les résistances, la détermination des objectifs, l'analyse du processus d'informatisation des données et la philosophie d'implantation.

À la fin de l'été 1985, le comité informatique du service social de l'Hôpital Sainte-Justine avait produit une grille d'évaluation familiale et sociale et déterminé six indices psychosociaux à mettre au dossier médical informatisé du patient. Depuis le début de 1986, la grille a été 
rodée, corrigée et adaptée au fur et à mesure de son utilisation dans la pratique. Le logiciel a été complété et sa présentation entièrement remaniée.

Dans le présent article, nous procéderons à la description et à l'analyse de ce matériel (deuxième version) puis nous aborderons la question de notre responsabilité dans l'intégration de ce logiciel professionnel dans les plans de développement de l'informatique des deux établissements auxquels nous appartenons.

Nous désirons auparavant rappeler que le logiciel a été créé spécifiquement en fonction de la pratique en milieu médical pédiatrique et il vise un objectif de participation à une médecine globale informatisée. Nous voulons en arriver à ce que l'équipe multidisciplinaire puisse « instantanément avoir à l'écran un portrait global de l'état actuel du patient ainsi que de sa situation familiale et sociale. De même, l'écran pourrait faire voir l'évolution longitudinale du patient et de sa famille ${ }^{3}$.

\section{Logiciel d'intervention familiale et sociale}

Le logiciel se compose d'une grille d'intervention familiale et sociale et d'une série de tests remplis par les parents. Ces tests sont facultatifs; ils sont utilisés au bon jugement du clinicien selon leur pertinence en regard de la problématique psychosociale.

\section{Contenu}

La grille d'intervention constitue la partie principale du logiciel. Elle traduit l'essentiel de l'activité professionnelle du travailleur social dans le milieu médical soit : l'évaluation familiale et sociale et le plan d'intervention psychosociale.

\section{Évaluation}

L'ensemble des éléments de l'évaluation ont été classifiés et regroupés en sept thèmes que nous avons appelés "indices psychosociaux ", selon une logique et un processus de classification exposés dans l'article précédent. Ce sont ces sept «indices psychosociaux» qui apparaissent dans un premier temps au dossier informatisé du patient. Ils forment un continuum: cinq indices évaluatifs $\rightarrow$ pronostic $\rightarrow$ orientation (tableau 1). 


\section{TABLEAU 1}

\section{Indices psychosociaux}

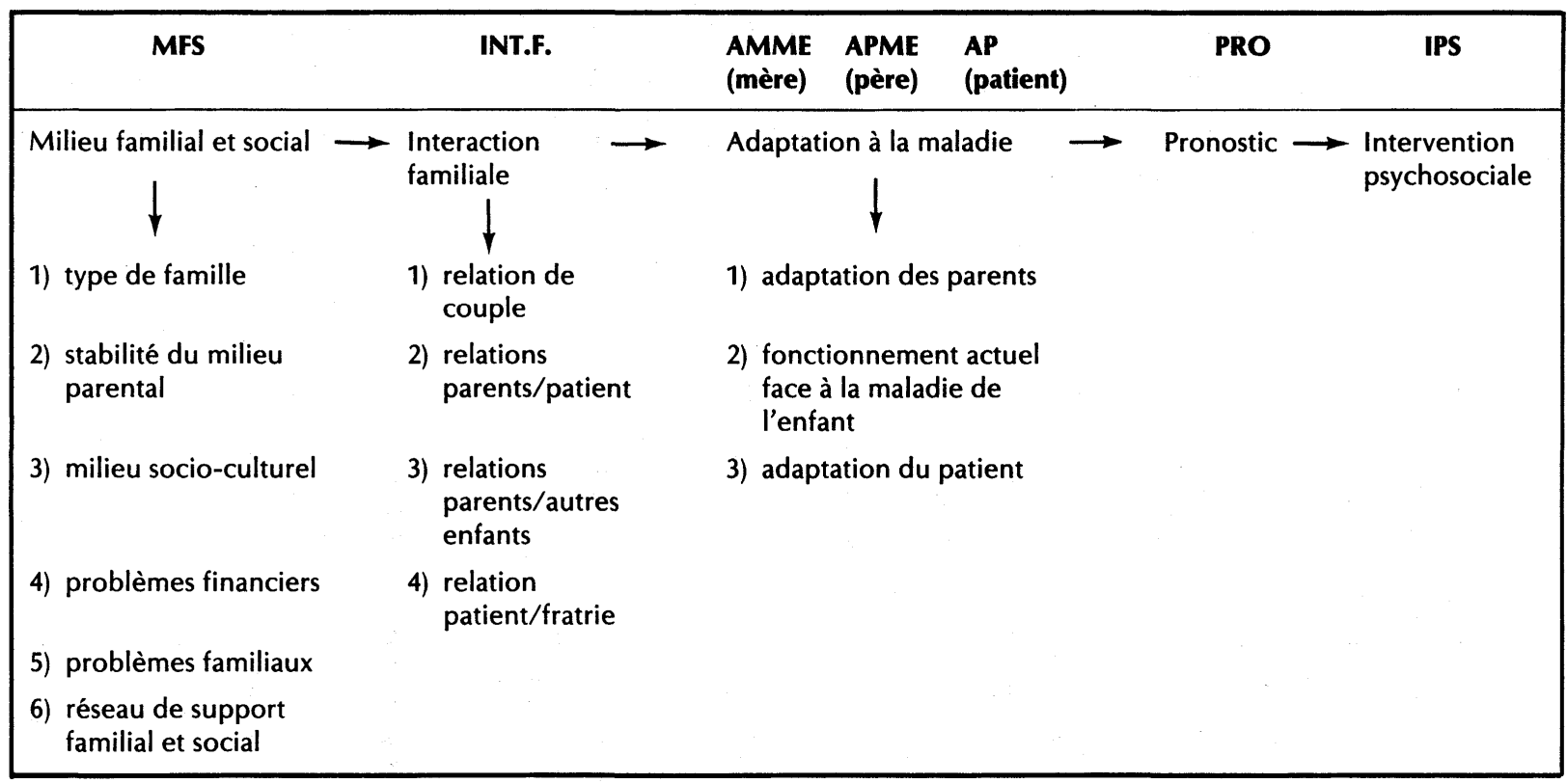




\section{Indices psychosociaux}

Chaque indice regroupe des sous-catégories d'éléments d'évaluation ; par exemple, « milieu familial et social » (MFS) comprend l'ensemble des ressources familiales et sociales; ce sont des données factuelles: type de famille, stabilité du milieu, réseau de support, etc. Le second groupe "interaction familiale" (INT.F.) a été dissocié du premier car ce sont des données dynamiques qui actualisent le groupe précédent en rapport avec l'indice suivant : " adaptation à la maladie " (tableau 1).

Les données psychosociales non factuelles de la première grille d'évaluation ont été, en cours de rodage, jugées trop globalisantes par le comité. Elles ont été reprises et détaillées dans leurs éléments constitutifs les plus significatifs dans la perspective de la problématique médicale. Ainsi, sous le thème "interaction familiale», la cote unique globale qui caractérisait la "relation de couple» a été remplacée par cinq cotes mesurant cinq sous-dimensions : la vie affective, la communication, la prise de décision, le partage des responsabilités et les activités de couple (tableau 2).

\section{Échelle d'appréciation}

Dans la première version de la grille d'évaluation, l'échelle d'appréciation se traduisait en termes de plus ou moins grande satisfaction. Pour cette deuxième version, nous avons adopté l'échelle utilisée par Skinner et Steinhauer ${ }^{4}$, laquelle correspondait davantage à notre philosophie d'intervention: chaque élément d'observation est évalué en termes de force et de faiblesse et coté de 1 à 5 . Les points faibles et les points d'appui à l'intervention sont ainsi simultanément mis en évidence. Cette échelle a été testée depuis et est reconnue dans onze milieux psychiatriques utilisant l'approche familiale ${ }^{5,6}$.

\section{Tests et questionnaires}

Les tests et questionnaires inclus dans le logiciel - et qui s'intègrent aux éléments de l'évaluation clinique - apportent une autre dimension à l'évaluation : la participation des personnes concernées à la définition de leur situation (tableau 3). Ces tests et questionnaires ont été sélectionnés d'après notre expérience clinique pour leur pertinence et leur valeur significative. Ils ont tous été expérimentés et plusieurs d'entre eux ont été évalués scientifiquement. Outre l'instrument de Skinner et Steinhauer déjà mentionné, le logiciel inclut ceux de Locke et Wallace $7,8,9$, de Moos et Moos ${ }^{10}$ et de Stein et Riessman ${ }^{11}$. Ils peuvent être remplis 
TABLEAU 2

Interaction familiale (INT.F.)

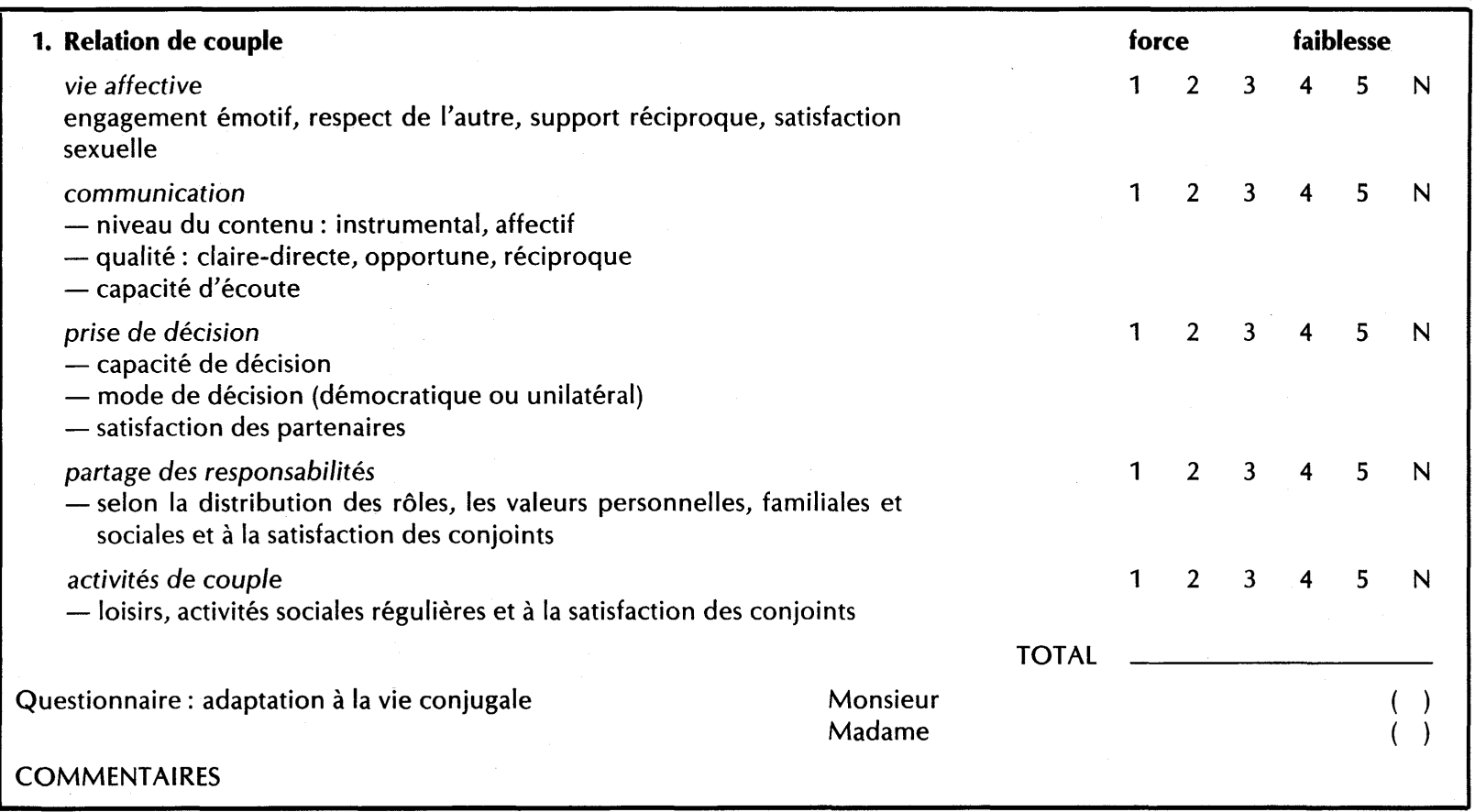


par les parents en quelques minutes et leurs données sont comptabilisées dans l'évaluation globale.

Enfin, pour personnaliser davantage le dossier, une section est réservée aux commentaires du professionnel à la fin de chacun des principaux points de l'évaluation. Le pronostic et l'orientation prévue (besoin ou non d'intervention, prise en charge ou transfert du dossier à un autre organisme), suivent les données évaluatives.

\section{Plan d'intervention}

Le plan d'intervention précise les objectifs poursuivis et les types d'intervention prévus : entrevues, placement, ressources, collaboration avec d'autres organismes. Le professionnel peut aussi faire les ajouts ou précisions qu'il juge appropriés.

\section{Rapport d'évolution}

La même grille d'évaluation peut servir pour le rapport d'évolution puisque celui-ci est basé sur les mêmes éléments évaluatifs. Ce qui

\section{TABLEAU 3}

\section{Tests \& questionnaires}

1. Niveau de stress et réseau de support social Department of Mental Health, California. Traduit par Régis Blais

2. Questionnaire d'adaptation à la vie conjugale (Marital Adjustment Test) Locke-Wallace Traduit par Jean-Marie Boisvert

3. Impact de la maladie sur la famille (Impact on Family Scale) Stein et Riessman Copyright 1978. Traduit par Dr Barry Pless

4. Échelle de l'environnement familial (Family Environment Scale) Rudolph Moos Copyright 1974. Traduit par René Forget

5. Renseignements supplémentaires sur la situation globale et l'aide souhaitée Françoise Turgeon-Krawczuk, 1985 
importe, pour la personne qui consulte le dossier, c'est la connaissance de la situation actuelle du patient. La comparaison des données dans le temps fournit une réponse sur l'évolution de la situation familiale.

\section{Présentation graphique du logiciel}

Nous avons voulu fournir au professionnel qui se sert du logiciel un outil simple d'accès et d'utilisation. La classification et la synthétisation logique du contenu centré sur la pratique courante ont été l'objet premier de nos préoccupations. La présentation graphique se devait de respecter aussi cet objectif : mise en page reflétant, au premier coup d'œil, l'ensemble d'un indice psychosocial ou d'un sous-élément d'évaluation; cote facile à repérer et à remplir; résultats rapidement visualisés.

Nous avons placé, à gauche de chaque page, les éléments de l'évaluation ou du plan d'intervention avec les explications concrètes des éléments sous-jacents et, à droite, les cotes à encercler. À la page 10 du logiciel, par exemple, se retrouvent ensemble, de façon globale et détaillée: l'adaptabilité de chacun des parents, et leur adaptation effective à la maladie de leur enfant (tableau 4). Ailleurs sont aussi présentés, sur une même page, les résultats finaux obtenus aux cinq indices d'évaluation, de même que le pronostic et l'orientation.

\section{Un logiciel adaptable}

Même s'il a été conçu en fonction d'une pratique en milieu médical pédiatrique, nous croyons que ce logiciel peut facilement être adapté à d'autres milieux: services sociaux médicaux pour adultes, services scolaires, centres locaux de services communautaires (C.L.S.C.), centres d'accueil, etc. Il s'agit, pour les professionnels responsables, d'ajuster les données en fonction de leur clientèle, de la problématique concernée et de leur utilisation du dossier social. Le client/enfant devient alors le client/adulte, enfant ou adulte handicapé, élève, bénéficiaire ou encore le client identifié. Des éléments sont ajoutés, d'autres retranchés parce que non appropriés. Le logiciel sert ainsi de base à une élaboration différente, collée à la réalité professionnelle de chaque problématique.

L'ensemble du logiciel est destiné à être informatisé, c'est-à-dire traduit en langage machine. Les données écrites ne se trouvent pas pour autant éliminées de notre pratique quotidienne. La grille d'évaluation 


\section{TABLEAU 4}

Adaptation à la maladie de l'enfant (mère : AMME père : APME)

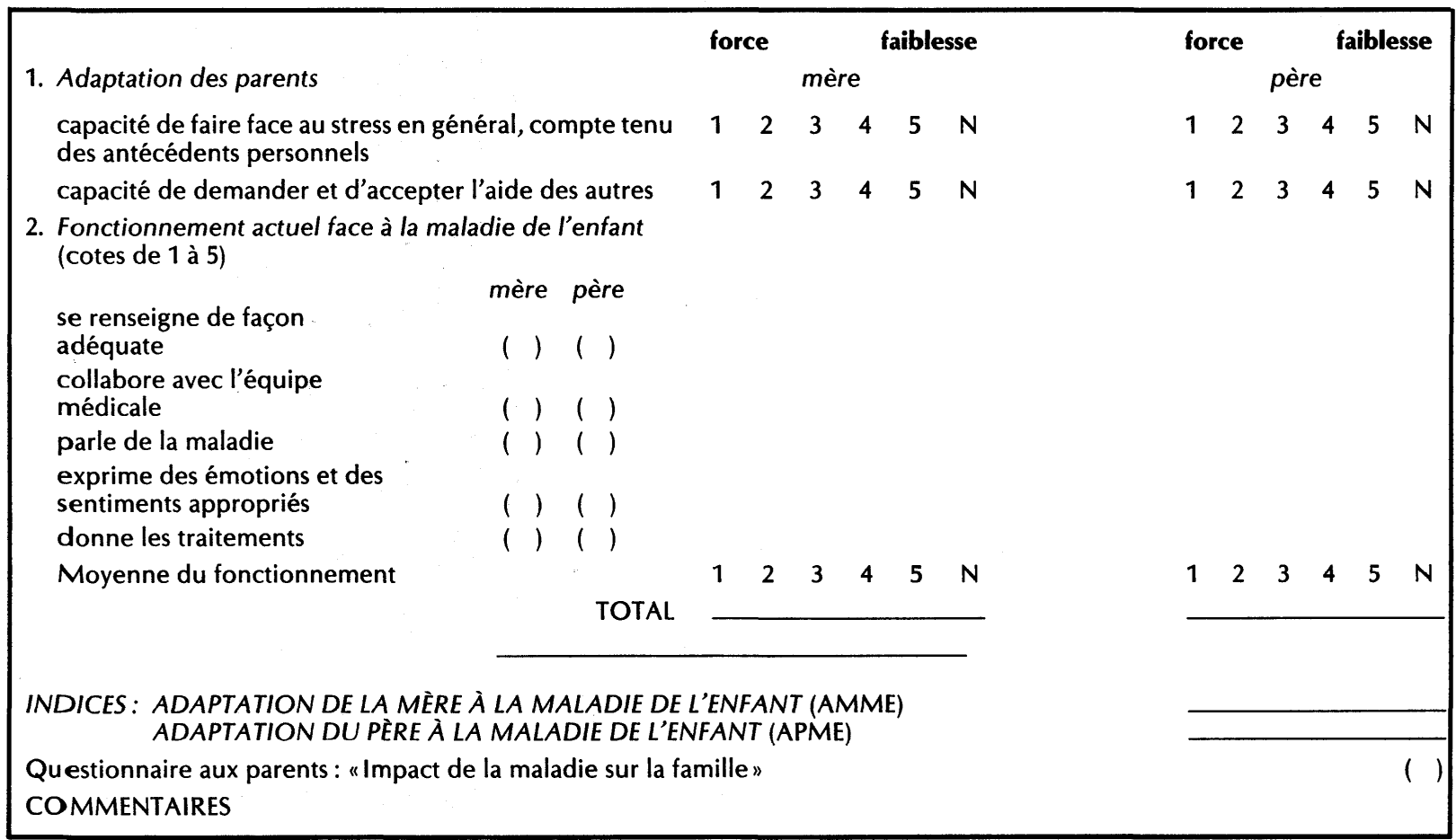


et les tests pourraient aussi être utilisés tels quels dans les dossiers. La grille réduit le temps consacré à la rédaction des dossiers et fournit un ensemble de données organisées et uniformisées.

\title{
La responsabilité du clinicien dans le processus d'implantation
}

\begin{abstract}
"La créativité, c'est imaginer de nouvelles choses. L'innovation, c'est faire de nouvelles choses. Une nouvelle idée-force peut circuler sans être utilisée dans une entreprise pendant des années, non parce qu'on ne reconnaît pas ses mérites, mais parce que personne n'a assumé la responsabilité de passer de la théorie à la pratique. Les idées sont inutiles si elles ne sont pas utilisées... Ceux qui ont le savoir-faire, l'énergie, l'audace et la persévérance nécessaires pour mettre ces idées en pratique sont rares. »12
\end{abstract}

L'intérêt soulevé par notre expérience d'implantation de l'informatique en service social clinique nous amène à élaborer davantage sur l'esprit qui anime notre démarche. Nous avons assumé une responsabilité professionnelle, celle de produire un logiciel de qualité. Notre effort ne s'arrête pas là, car notre responsabilité, même partielle, s'étend à tout le processus d'implantation.

Établissant un parallèle avec ce qui se passe dans les meilleures entreprises américaines à l'occasion du lancement d'un nouveau produit ${ }^{13}$, nous remarquons que le rôle du "champion volontaire" se révèle primordial dans la réussite de toute innovation. D'après les auteurs déjà cités, le produit gagnant est toujours soutenu par un individu qui y croit intensément - un pragmatique qui va bien au-delà des procédures officielles dans sa détermination à mettre son idée en pratique. Il a un besoin essentiel d'être appuyé dans sa démarche par certains cadres et autres "parrains" qui le protègent de la bureaucratie et qui assurent avec lui le succès du projet.

Le déroulement du processus d'informatisation des données psychosociales est conditionné par des facteurs humains et organisationnels qui créent souvent des résistances imprévues. Nous ferons l'analyse rétrospective de notre démarche au cours de la dernière année, en brossant tout d'abord un tableau de la situation telle qu'elle se présente sur le plan administratif. En deuxième lieu, nous identifiant au rôle du "champion volontaire " et nous basant, en partie, sur les théories émises par Peters et Waterman, nous énumérerons quelques dispositifs d'action utilisés de même que certains stimulants de notre action. 


\section{Situation administrative et problèmes présentés}

La double appartenance au centre hospitalier et au centre de services sociaux (C.S.S.) crée une ambiguïté dans notre situation de travail : notre action professionnelle se déroule au sein d'un établissement mais, administrativement, nous relevons d'un autre. Chacun d'eux possède une philosophie, des lois, des règles, des procédures et une gestion qui lui sont propres. Le dossier du service social, par exemple, fait partie intégrante du dossier médical de l'hôpital, mais il appartient légalement, aussi, au C.S.S.

Dans une démarche innovatrice, une situation de double appartenance a évidemment pour effet de multiplier les actions à entreprendre auprès des administrations : information, autorisation, clarification des enjeux, etc. De plus, chacun des établissements auxquels nous appartenons avait déjà établi son plan informatique: l'informatisation des dossiers sociaux ne faisait pas partie de leurs priorités.

Une autre difficulté était reliée à notre propre statut à l'intérieur du C.S.S. Placé au dernier échelon de la structure organisationnelle, "l'intervenant a peu de pouvoir décisionnel " ${ }^{14}$. Son rôle est bien défini et son rendement mesuré en termes quantitatifs: dossiers ouverts, nombre d'entrevues, etc. Le rendement qualitatif, plus difficilement mesurable, s'avère moins « rentable » pour un service ou un établissement. Approches préventives, efforts de changements structurels, publications, innovation professionnelle deviennent secondaires, une "denrée de luxe", surtout dans le contexte actuel de restrictions budgétaires.

Ces problèmes d'introduction d'une nouvelle application informatique, bien que redoutables, n'ont pas suffi à bloquer notre élan. "Comme en présence de tout changement, les comportements vont dépendre à la fois des motivations individuelles et des possibilités d'action offertes à chacun par le contexte organisationnel "15. Nous présenterons donc maintenant quelques moyens d'action qui nous ont été fort utiles.

\section{Un groupe d'intervention ad hoc: le comité " informatique " du service social}

Constitué d'un petit nombre de volontaires qui croient à ce qu'ils font, tourné vers l'accomplissement d'une tâche précise dans un laps de temps limité, ce groupe jouit d'une autonomie professionnelle relative stimulant l'action. Au sein d'une institution lourde, bureaucratique et très réglementée, un tel groupe risquerait fort d'être enfermé dans le labyrinthe préexistant et d'être paralysé. Lorsque le contexte s'y prête, 
comme c'est le cas au service social de Sainte-Justine, le groupe devient un outil très efficace pour mettre en ouvre les solutions et pour stimuler les efforts de tous, dans un système qui demeure hiérarchique.

Il faut souligner toutefois que la priorité du service social est l'aide directe à la clientèle. Le travail du comité n'a donc pas été reconnu comme prioritaire et la tâche clinique des membres n'a pas été allégée. Pour mener à bien l'entreprise, il a fallu y consacrer beaucoup de temps personnel, faire preuve d'engagement et d'une grande détermination.

\section{Le réseau de pairs (networking) : un moyen d'échange et de communication}

Peters et Waterman ${ }^{16}$ parlent beaucoup des structures d'organisation souples, d'autonomie maximale pour les individus, d'expérimentation régulière, de feedback mettant l'accent sur le positif, et de réseaux sociaux solides. Pour eux, le parti pris de l'action, y compris l'expérimentation, implique avant tout une communication intensive, extrêmement régulière, informelle et ouverte. Cette politique des contacts opportuns amène une certaine fluidité dans l'organisation, ce que l'appareil bureaucratique tolère mal. La léthargie et l'inertie qui résultent d'une communication trop formelle, pauvre et routinière, paralysent beaucoup d'entreprises et de projets.

Dans son livre Megatrends, Naisbitt ${ }^{17}$ analyse une nouvelle tendance qui transforme notre vie et surtout nos relations de travail : la remise en question de la structure hiérarchique et de son système de communication trop rigide au profit d'une interaction plus personnelle, plus démocratique, plus respectueuse des qualités intrinsèques de chacun et beaucoup plus efficace. Pour maintenir notre motivation et faire avancer notre dossier, nous avons fait une grande utilisation du networking, créant et cultivant nos contacts avec les personnes intéressées aux mêmes expériences que nous, partageant idées, informations et ressources, établissant ainsi un réseau de support.

\section{Le support de l'extérieur : un moyen de pression pour le changement}

La publication et la diffusion d'un premier article faisant connaître notre démarche ont suscité beaucoup d'intérêt dans le milieu des services sociaux québécois. Ces marques concrètes d'appréciation et de support venant de professionnels et d'organismes extérieurs ont servi à renforcer notre position face à nos instances administratives. 
Grâce à l'utilisation consciente de ce puissant moyen de pression, l'innovation que nous proposions a reçu un meilleur accueil, et les possibilités d'action et d'implantation ont été améliorées.

\section{La collaboration avec la profession médicale : une alliance avec un pouvoir établi}

L'évolution dynamique du service social dans un important centre hospitalier universitaire ne peut être assurée sans une participation étroite au programme d'informatisation du dossier médical. C'est d'ailleurs sur l'insistance de quelques médecins, et dans le cadre de la pratique de la médecine globale, que nous avons commencé notre propre démarche d'informatisation.

Le rôle du pouvoir dans le processus d'informatisation a été analysé, entre autres par Dupuy ${ }^{18}$. La possibilité qu'ont les médecins de passer à l'action est reliée au pouvoir tiré de leur statut hiérarchique formel dans l'hôpital, de même qu'à leur influence fondée sur le prestige et l'appartenance à une puissante association professionnelle. Leur appui constant s'est révélé essentiel au succès de notre projet : par leur intermédiaire, l'accès à des fonds privés a permis de retenir les services de consultants en informatique pour assurer la qualité du produit; ils ont exercé des pressions efficaces sur l'administration pour obtenir les terminaux essentiels à l'expérience; ils ont supporté notre projet et diffusé nos idées lors de diverses communications, en particulier lors de présentations scientifiques.

Plusieurs travailleurs sociaux ont une crainte bien ancrée d'un rapprochement avec les médecins. On s'inquiète de la mise en tutelle de notre profession : pour beaucoup d'entre nous, collaboration signifie annihilation. Nous pouvons témoigner que, loin de nous affaiblir, une pratique vraiment intégrée nous enrichit et nous assure le respect de la profession médicale.

\section{La collaboration avec l'Université : une alliance qui renforce le « moi " professionnel}

La recherche et la publication sont des moyens privilégiés pour assurer l'augmentation des connaissances et la diffusion de l'expertise. Ce sont aussi des stimulants de l'action : en s'engageant dans ce domaine, on se compromet, on ne peut plus reculer, le mouvement en avant est assuré. De plus, les subventions à la recherche et les facilités de publication accroissent le sentiment de la valeur de ce que l'on fait et encouragent la poursuite de l'effort ${ }^{19}$. 


\section{La reconnaissance du droit d'auteur : un support à la motivation et à la productivité}

Peters et Waterman ${ }^{20}$ consacrent une partie de leur livre : Le prix de l'excellence, à la motivation des employés, soulignant qu'il est nécessaire de la soutenir par des signes de reconnaissance positifs. Même si dans les meilleures entreprises il existe de "somptueux systèmes de stimulants financiers", on trouve aussi un nombre considérable de stimulants non pécuniaires. Ce souci d'améliorer la productivité en soutenant de façon positive la motivation des employés se retrouve dans la politique actuelle de rénovation de l'administration publique québécoise. Le Conseil du Trésor cherche les moyens d'améliorer les services à la clientèle et souligne le point essentiel de la motivation des employés : " Le manque de reconnaissance du rendement exceptionnel des employés risque de provoquer une sorte de désaffection et de nivellement par le bas qui a forcément un impact sur la productivité " ${ }^{21}$. Dans le même ordre d'idée, la tendance, ou du moins la tentation actuelle de nier le droit d'auteur d'un employé qui crée une ouvre originale et d'attribuer ce droit à l'employeur, constitue une bonne façon de démobiliser le personnel. Nous faisons référence ici, non pas aux droits pécuniaires, mais plutôt au droit moral qui permet de revendiquer la paternité de l'œuvre originale. Pour nous, la reconnaissance du droit d'auteur n'est pas seulement un stimulant de l'action, elle touche la notion de justice relativement à la production d'un logiciel qui sera, du moins nous l'espérons, largement utilisé 22 .

Après réflexion et consultation, nous avons donc décidé d'obtenir le droit d'auteur pour notre logiciel, imitant d'ailleurs en cela bon nombre de professionnels nord-américains.

\section{Conclusion $^{23}$}

Créer un logiciel professionnel constitue une démarche dont les exigences d'implication se sont révélées au-delà de nos prévisions. Notre conviction d'agir selon une nécessité professionnelle, dans le contexte de l'évolution du milieu de pratique et de la société actuelle, nous a soutenues. Les répercussions positives dans le réseau des services sociaux ainsi que le support du milieu universitaire ont stimulé notre action créative et renforcé notre pouvoir de négociation. 


\section{Notes et références}

1 Y. LeCLeRC, "Demain l'an 2000 : les programmes introuvables", La Presse, 8 mars 1986 : D-2.

2 F. Turgeon-Krawczuk et C. Lalande-Gendreau, "L'informatique en service social: une application à la pratique professionnelle en milieu médical ", Intervention, $\mathrm{n}^{\circ}$ 73, 1986 : 37-49.

3 Id., p. 40.

4 H. Skinner, P. Steinhauer et J. Santa-Barbara, "The family assessment measure", Canadian Journal of Community Mental Health, vol. 2, n० 2, 1983 : 91-105.

5 H. SKInNer et P. STEINHAUER, "Synopsis of projects using FAM III ", texte remis au Workshop on Family Assessment, Montréal, avril 1986.

6 H. SKinner et P. Steinhauer, "References ", texte remis au Workshop on Family Assessment, Montréal, avril 1986.

7 H.J. LOCKE et K.M. WALLACE, "Short marital adjustment and prediction tests : Their reliability and validity ", Marriage and Family Living, vol. 21, n० 2, 1959 : 251-255.

${ }^{8}$ D.G. Cross et C.F. Sharpley, "The Locke-Wallace marital adjustment test reconsidered: Some psychometric findings as regards to its reliability and factorial validity ", Educational and Psychosocial Measurement, vol. 41, no 4, 1981: 1303-1306.

9 G.L. HANSEN, "Marital adjustment and conventionalization : A reexamination ", Journal of Marriage and the Family, vol. 43, no 4, 1981 : 855-863.

10 R. Moos et B. Moos, Family Environment Scale Manual, Palo Alto (Ca.), Consulting Psychologists Press, 1981.

11 R. Stein et C. ReISSMAN, "The development of an impact on family scale: Preliminary findings ", Medical Care, vol. 18, no 4, 1980: 465-472.

12 T. Peters et R. Waterman, Le prix de l'excellence, Paris, Inter-Éditions, 1983, p. 216.

13 Id.

14 C. Lalande-Gendreau et M. Rinfret-Raynor, "Les motivations des travailleurs sociaux dans les centres de services sociaux du Québec ", Intervention, n 63, hiver 1982, p. 43.

15 Y. DupuY, "Les obstacles humains à l'informatisation", Revue française de gestion, no 43, 1983, p. 78.

16 T. Peters et R. Waterman, op. cit.

17 J. NAISBITT, Megatrends, New York, Warner Books, 1982.

18 Y. DUPUY, op. cit.

19 La collaboration active aux travaux de recherche de deux professeurs de l'École de service social de l'Université Laval a été un élément dynamique de notre démarche d'informatisation.

20 T. Peters et R. Waterman, op. cit.

21 G. LESAGE, "Contrer les effets principaux du vieillissement : un plan de rénovation de l'administration publique québécoise", Le Devoir, 19 juillet 1986, p. A-1. 
22 La loi sur le droit d'auteur, qui date de 1924, doit être bientôt changée. Le projet de loi (1984) se propose d'apporter une protection particulière aux programmes informatiques. II pose aussi la question : qui devrait détenir le droit d'auteur sur les œuvres créées par des employés dans l'exercice de leurs fonctions? Le public est invité à se prononcer là-dessus.

"Pour le Québec, toutes les œuvres de l'esprit doivent être protégées et recevoir la même protection, qu'elles soient de nature informatique ou que l'informatique serve de support original à l'œuvre ou dérive d'une œuvre préexistante." (Clément RıCHARD, "Position du Gouvernement du Québec à propos du Livre blanc sur les droits d'auteur ", Gouvernement du Québec, Ministère des affaires culturelles, 1985, p. 6.)

Plus loin, le document ajoute : "Dans le cas des œuvres créées par un employé du Gouvernement ou d'une entreprise privée, le Québec recommande que le bénéficiaire du droit soit l'employé, sous réserve de la reconnaissance législative à l'employeur d'une licence exclusive d'utilisation de l'œuvre dans le cadre de son activité régulière." (P. 8.)

23 Article accepté pour publication en juillet 1987. 